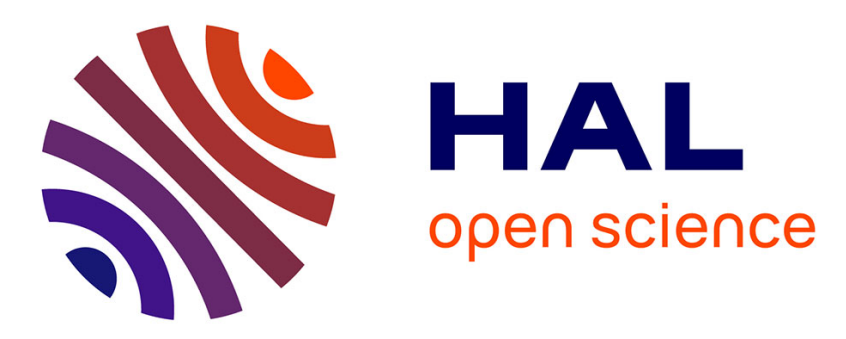

\title{
1D Cahn-Hilliard dynamics: Transition modes and stability criteria
}

\author{
Mahdi Mcheik, Simon Villain-Guillot
}

\section{To cite this version:}

Mahdi Mcheik, Simon Villain-Guillot. 1D Cahn-Hilliard dynamics: Transition modes and stability criteria. CCT15 (Conference on Chaos, Complexity and Transport 2015 ), Jun 2015, Marseille, France. 10.1142/9789813202740_0014. hal-02457581v2

\section{HAL Id: hal-02457581 \\ https://hal.science/hal-02457581v2}

Submitted on 4 Feb 2020

HAL is a multi-disciplinary open access archive for the deposit and dissemination of scientific research documents, whether they are published or not. The documents may come from teaching and research institutions in France or abroad, or from public or private research centers.
L'archive ouverte pluridisciplinaire HAL, est destinée au dépôt et à la diffusion de documents scientifiques de niveau recherche, publiés ou non, émanant des établissements d'enseignement et de recherche français ou étrangers, des laboratoires publics ou privés. 


\title{
1D Cahn-Hilliard dynamics :
}

\section{Transition modes and stability criteria}

\author{
Mahdi Mcheik, Simon Villain-Guillot \\ Laboratoire Ondes et Matière d'Aquitaine, Université de Bordeaux and \\ 351, cours de la Libération 33405 Talence Cedex, France*
}

\begin{abstract}
The Cahn-Hilliard-Oono equation describes the dynamics of phase separation process of copolymers alloys and other binary systems. This process is driven by the minimization of the free energy. However the lower energy state in rarely reached and only for particular initial states. Misbah and Politi have proposed a dynamical criterion which leads to final states that doesn't correspond to the minimization of free energy. In this article we investigate possible transition modes that may describe the dynamic of "segregation and coalescence" in those systems, in order to understand the difference between these criteria.
\end{abstract}

*Electronic address: simon.villain-guillot@u-bordeaux.fr, mahdi.mcheik@u-bordeaux.fr 


\section{INTRODUCTION}

In many systems such as melts of diblock copolymer, which are built by linking two hetero-polymers, the competition between opposite interactions with long and short range, may form spatially modulated patterns below a critical temperature (or Flory-Huggins parameter). Indeed, the interaction between homogeneous polymers is attractive while the interaction between heterogeneous polymers is repulsive and thus favors segregation ; the simplest dynamics after a quench tends to reduce the interfaces between homogeneous polymer domains so as to minimize the interfacial free energy. However, as diblock copolymers are attached to each other, the complete segregation cannot occur as for the usual liquid gas phase transition or in simple polymer blends, where domain sizes can become infinite. This frustration results in the formation of spacially periodic structures. The length of the phase modulation depends on the relative strength of the two competiting forces ; in other terms, the dynamics reduces the interfacial free energy until some finite value of wavelength for which the two forces counterbalance.

The Cahn-Hilliard-Oono's equation is particularly adapted to describe the dynamics in such system. It is a conservative modified diffusion equation for a scalar order parameter $u$ :

$$
\frac{\partial u}{\partial t}=\left(\nabla^{2} \frac{\delta F_{G L}(u)}{\delta u}\right)-\alpha u=\nabla^{2}\left(-u+u^{3}-\nabla^{2} u\right)-\alpha u
$$

The order parameter $u$ can correspond to a rescaled density of atoms or relative concentration of polymers, $-u+u^{3}$ derives from the Landau free energy which characterizes the short ranges repulsive interactions and the entropy of mixing, and the Oono's term $\alpha u$ describes the non-local long-range interaction which tends to homogenize the system and forbid the full segregation. The competition between the two parts of the dynamics results in the formation of a spatially peridically modulated state.

This conservative dynamics derives from the free energy :

$$
\mathcal{F}(u(r))=\int F_{G L} \mathrm{~d} r+\int F_{\text {int }} \mathrm{d} r
$$

$F_{G L}$ is the Ginzburg-Landau free energy density $F_{G L}=\frac{1}{2}(\nabla u(r))^{2}+\frac{-1}{2} u^{2}(r)+\frac{1}{4} u^{4}(r)$ $F_{\text {int }}$ is the Leibler free energy density (corresponding to Oono's non-local interaction 
term in the dynamics),

$$
F_{\text {int }}=\alpha \int u\left(r^{\prime}\right) g\left(r^{\prime}, r\right) u(r) \mathrm{d} r^{\prime} .
$$

where $g$ is the kernel of the long range interaction term and is such that $\nabla^{2} g\left(r^{\prime}, r\right)=-\delta\left(r^{\prime}-r\right)$.

This dynamics is driven by the minimization of the free energy (1). However direct simulations for one dimension dynamics show that the final solutions are not unique and they depend on the initial states. Moreover, starting with initial random conditions, the associated final free energies don't correspond to the lower energy state. This lower energy is found numerically only when $\alpha$ is large (Swift-Hohenberg limit). Otherwise, for large 1D systems, and starting with initial random conditions, numerical simulations show that many different domain sizes may coexist, due to the very slow dynamics. Therefore it is important to study the distribution of those solutions.

This paper is composed of three parts; in the first part we focus on different transition modes for coarsening, in the second part we show two criteria that we used to define stability and the last part is dedicated to the statical study of domain sizes of final solutions.

\section{TRANSITION MODE AT COARSENING STAGE}

In this section, we will focus on different transition modes that can affect the dynamics of the Cahn-Hilliard equation during the coarsening regime which follows the spinodal decomposition. During this last stage of the dynamics, we believe that the upper limit of the wave number $q$ of the steady-state solution results from the Ostwald ripening, i.e from the diffusion between domains. This process generates interface displacements in both directions, and leads to the creation of a profile of larger wavelength.

We have simulated the dynamics numerically, focusing on the question of the stability of stationary (or frozen) solutions obtained from direct simulation in small boxes. We have used the same pseudo-spectral algorithm used by M. Nicoli (IFABM4), as this method is known for its stability and accuracy. But we have used another criterion [6] to confirm if these solutions remain absolutely stable for larger boxes when perturbed by small amplitude 


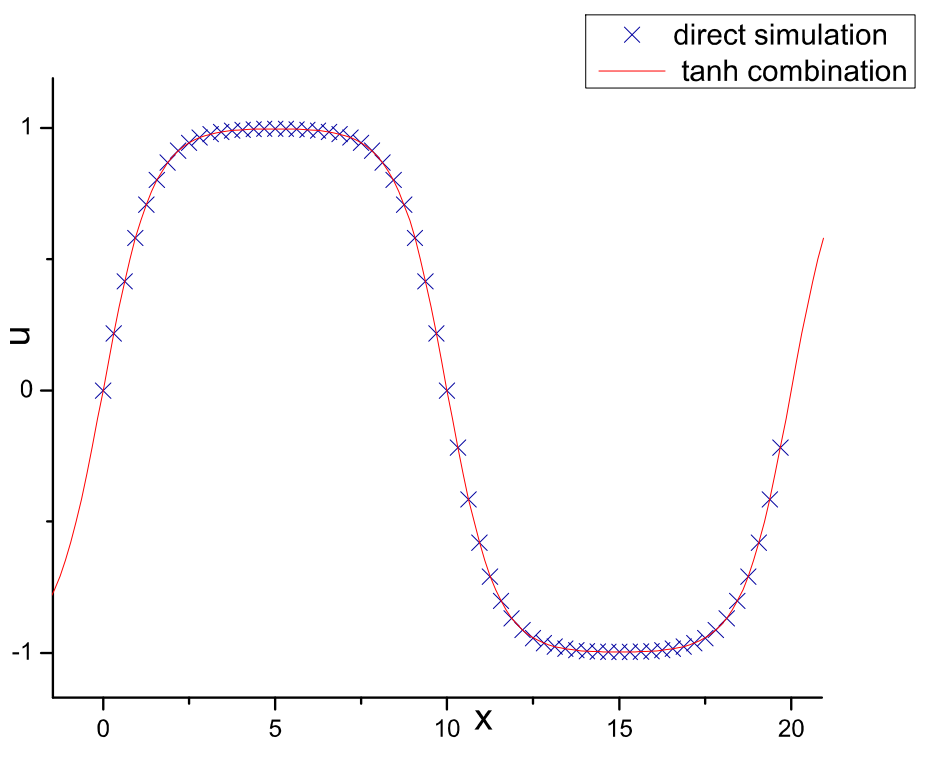

FIG. 1: In blue numerical solution of $\mathrm{CHO}$ equation starting from random initial state $\left(\alpha=10^{-4}\right.$ and $\lambda=20$ ), in red hyperbolic tangentes combination used to simulate the numerical solution.

noise, i.e. we have studied the evolution of perturbation superimposed above these frozen patterns replicated in larger commensurate boxes .

Starting from random initial conditions, or starting close to the expected solution, the stationary solutions of $\mathrm{CHO}$ equation obtained for long time simulation, are, in the case of small simulation box (typically smaller than 8 lambda $\mathrm{CH}$ ), close to elliptical sine $\mathrm{Sn}$ function. Therefore we make use of the expansion of Sn in terms of hyperbolic tangents, to simulate the process of coarsening by the motion of tanh interfaces.

$$
S n_{\lambda}(x)=\sum_{i}(-1)^{i} \tanh (s(x-i . \lambda / 2))
$$

where $s$ is extracted from the numerical simulation looking at the the tangent for small values of the order parameter $u(x)$ (see FIG 1 ) and $\lambda$ is the known wavelength.

The third parameter needed for our expansion is the amplitude that can be adjusted easily from the numerics.

The resulting profiles fit very closely the numerical results (as can be seen in the FIG 1), the errors (amplitude and tangent) for different value of $\alpha$ are less than $1 \%$ while error on energy is reasonably small for $\alpha<10^{-2}$ and $\lambda>2 \lambda_{C H}$. The coarsening process can then be 
simulated by looking solely at the motion of tanh interfaces whose only degree of freedom are the positions.
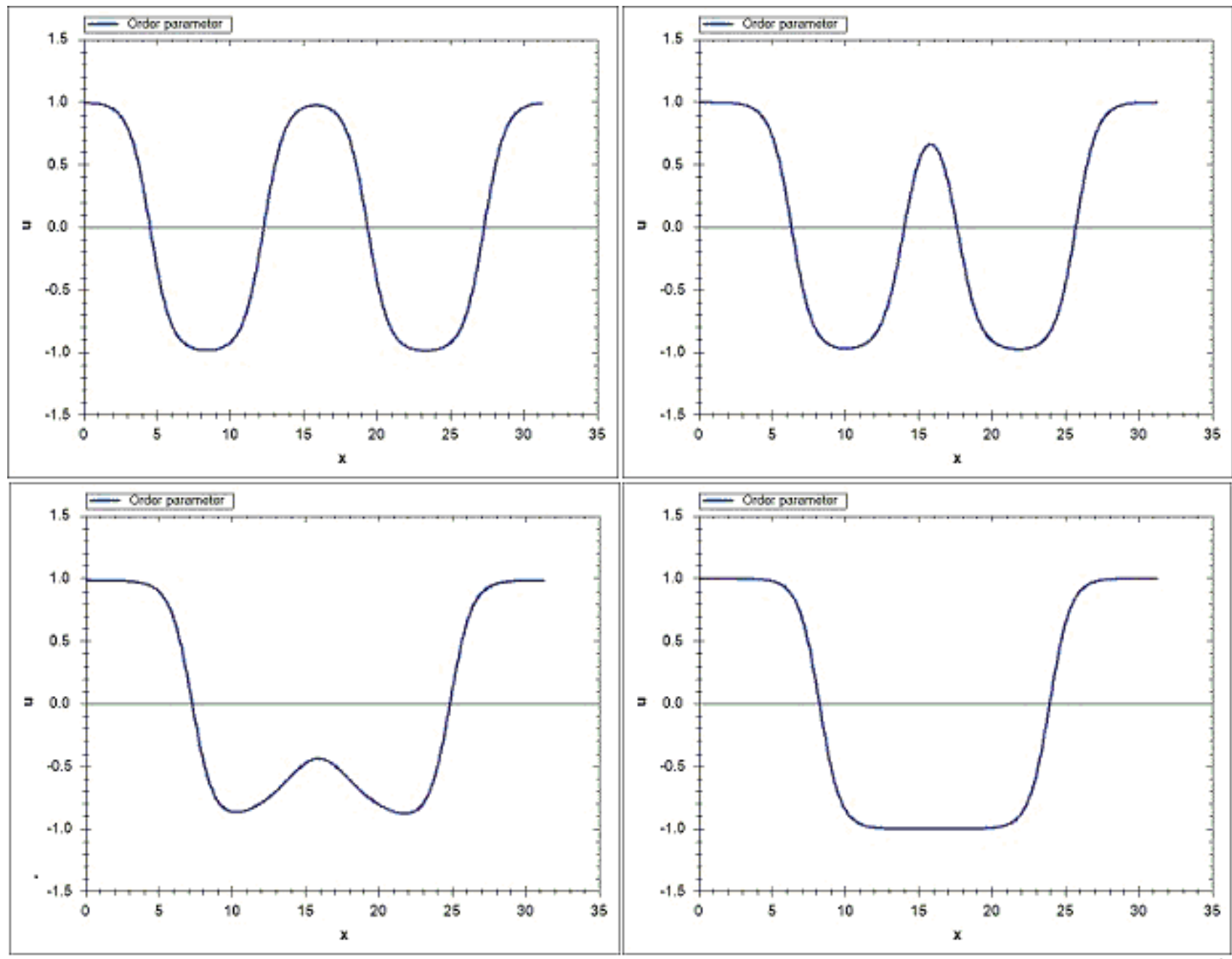

FIG. 2: This four pictures illustrate the dynamics extracted from direct numerical simulation of CHO equation acording transition mode " $2 \rightarrow 1$ ", when $\alpha=10^{-8}$

Figures 2 show one possible transition mode " $2 \rightarrow 1$ ", as seen in a simulation of $\mathrm{CHO}$ equation starting with 4 interfaces and wavelength 15, merge into a pattern of double wavelength. The two inner kinks vanish together in symmetrical way, while the outer kinks move to the center at the same velocity. Other modes were seen during visualizations of numerical simulations of CHO dynamics, for example " $3 \rightarrow 2$ ", " $5 \rightarrow 3$ " and some other can be obtained with this procedure such as " $5 \rightarrow 4$ "," $7 \rightarrow 6$ " and so on... 


\section{COALESCENCE AND MOTION}

One possible scenario for coarsening is the symmetric diffusion, where the transfer of matter between neighboring domains can be described in term of the motion of interfaces. This symmetric process naturally conserves matter. The dynamics of these tanh interfaces is indeed very close to the numerical simulation of the full CHO equation. The correlated motion of the tanh interfaces affects the shape (or parameters) of the entire profile, even if is the nearest interfaces are more impacted ; in that sense, hyperbolic tangent has a nonlocal effect on the entire profile. For example, the evolution of the tangent of the profile at $u(x)=0$ depends on the distance between interfaces ; or the amplitude increases with $\lambda$, this variation being more important for small $\lambda$ as we can notice in Fig 3.

The figures 3, 4 and 5 shows interface's motion that allow the merging respectively of 2 into 1, 3 into 2 , and 5 into 3 patterns.

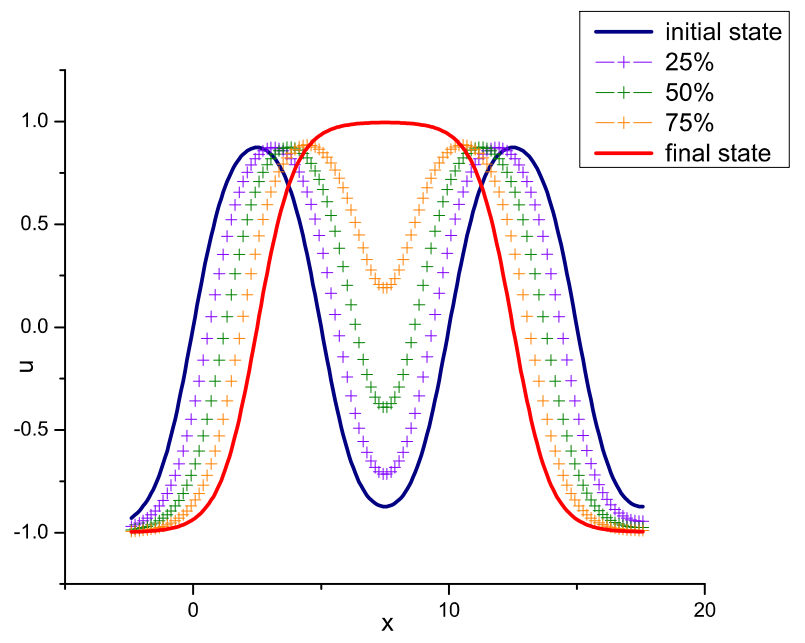

FIG. 3: Transition $2 \rightarrow 1$ : in blue initial state (wavelength 10 ), in red the final state (1 pattern of double wavelegnth), the discontinued line shows intermediate states

If $\alpha=0$, the stationary solution is known analytically to be the elliptical sine Sn. When interfaces start to move in order to create larger domains, the overall free energy decreases always monotonically during this process which can be parametrized by the displacement of the interfaces, from 0 to $\lambda_{\text {initial }} / 4$ in the process $" 2 \rightarrow 1$ ". 


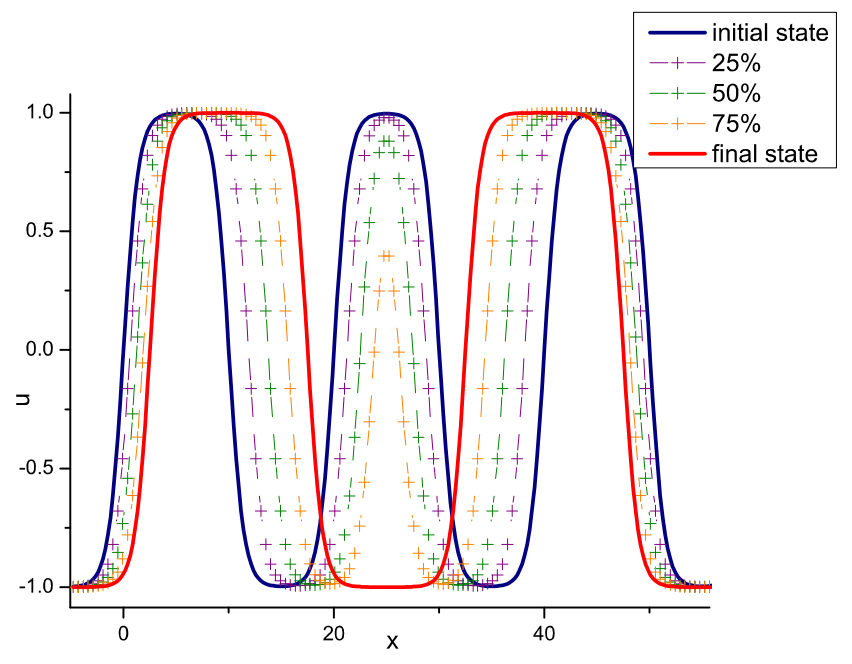

FIG. 4: Transition $3 \rightarrow 2$ : in blue initial state (wavelength 15 ), in red the final state (2 patterns of $3 \times 15 / 2$ wavelegnth), the discontinued line shows intermediate states

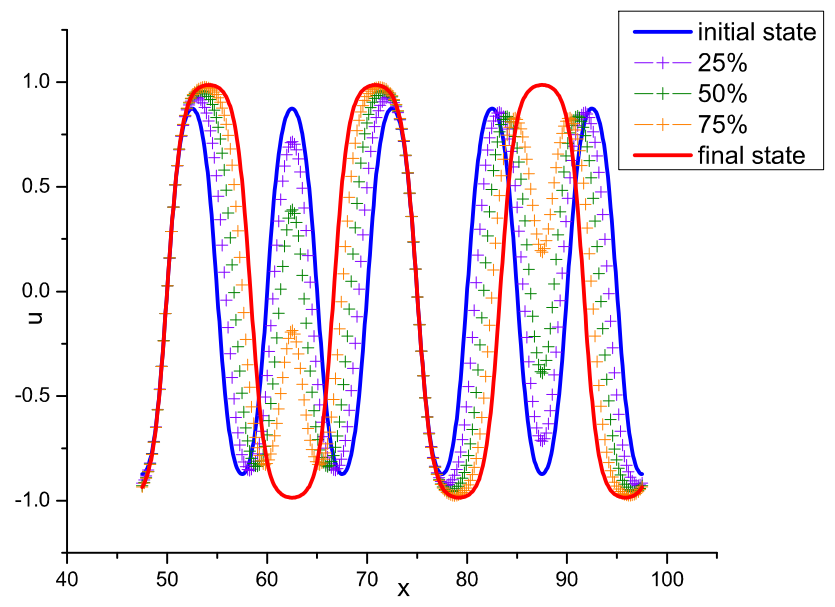

FIG. 5: Transition $5 \rightarrow 3$ : in blue initial state (wavelength 10 ), in red the final state (3 patterns of $5 \times 10 / 3$ wavelegnth), the discontinued line shows intermediate states

But when $\alpha$ is non zero, the evolution of the overall free energy is not anymore always deacreasing when domains coarsen. Indeed, for any non zero values of $\alpha$, we found that for large enough values of the wavelength, the free energy would increase with further displace- 

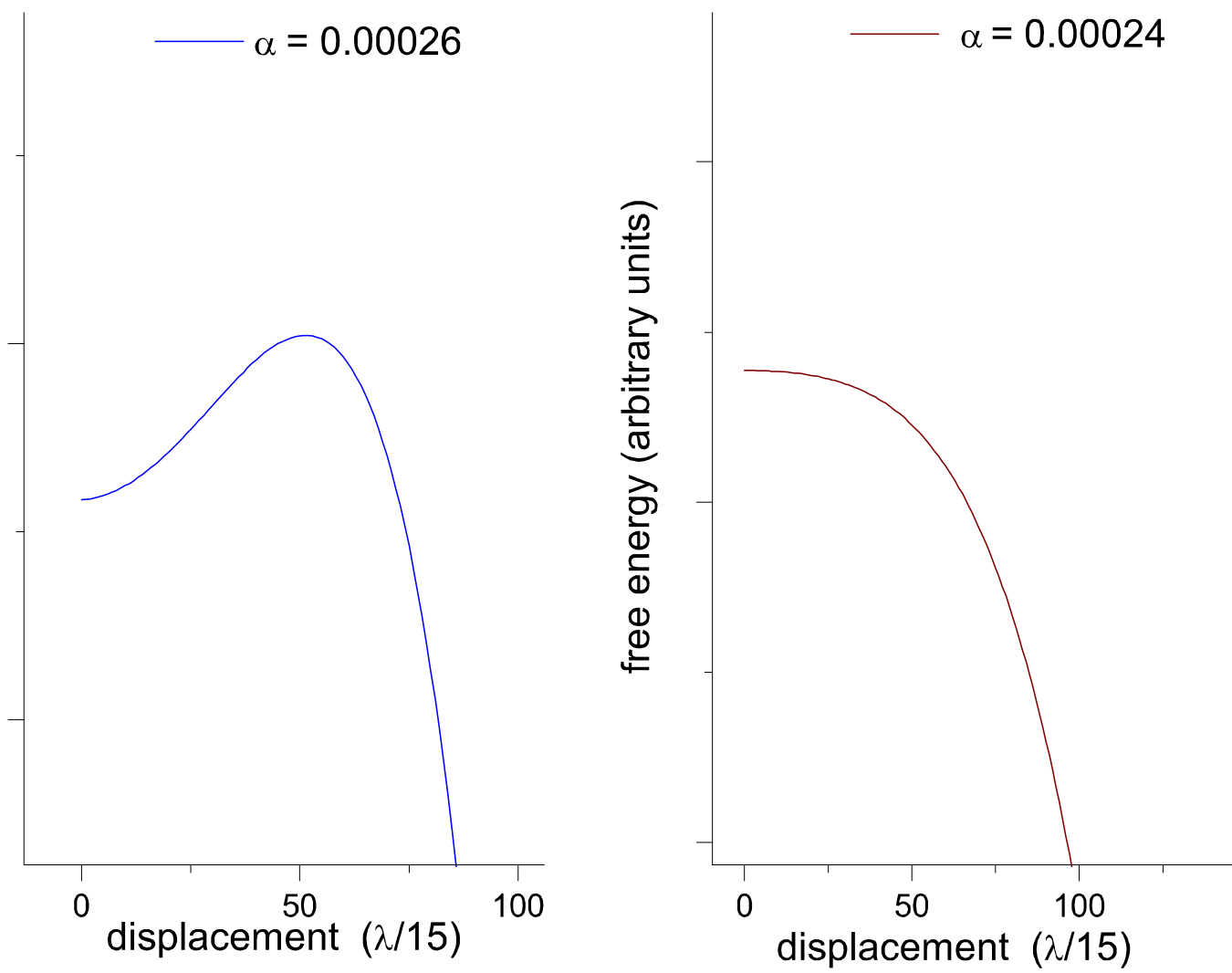

FIG. 6: Blue curve: a profile of free energy variation in term of displacement of interfaces where $\alpha=2.4 \times 10^{-4}$ and $\lambda=4 \pi$.

Red-Wine curve : an example where $\lambda=4 \pi$ and $\alpha=2.6 \times 10^{-4}$ is slightly greater than some critical value $\alpha_{c}$ for wchich the dynamics is interrupted. One can see in this case some kind of potential well that would stop the motion of the interfaces. This well becomes deeper for greater values of $\alpha>\alpha_{c}$.

ment of the interfaces along the " $2 \rightarrow 1$ " process. For $\lambda>\lambda_{c}(\alpha)$, the profile is thus stable with respect the studied coarsening motion of the interfaces. In other words when further segregation would cost energy, coarsening dynamics is interrupted [2] and the segregation remains partial.

In picture 6 we see that for $\lambda=4 \pi$, the free energy decreases as function of displacement 
when $\alpha \leq 2.4 \times 10^{-4}$, but a small potential well appears for bigger values $\left(\alpha=2.6 \times 10^{-4}\right)$ so we consider that profiles of periods $4 \pi$ are instable if $\alpha \leq 2.4 \times 10^{-4}$. In Figure 7 we illustrate the thresholds of stable solutions of given values of $\alpha$ for different scenarios of symmetrical segregations $(2 \rightarrow 1,3 \rightarrow 2$..)

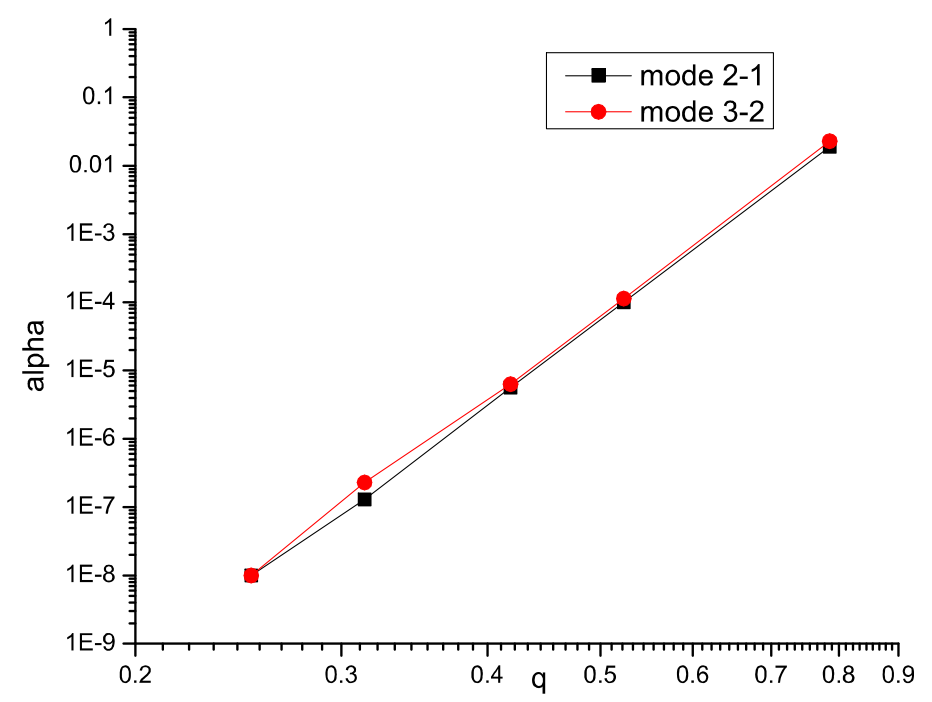

FIG. 7: In red, the stable wavelengths associated with the transition mode 2-1, in black, the wavelengths associated to mode 3-2

These 2 curves are almost identical ; and we obtain identical behaviors for other modes like merging 7 patterns into 6, 9 into 8 , and even 5 into 3 . Therefore, we can ensure that the upper limit of $q$ is given by the symmetric diffusion, found analytically by Misbah and Torcini [5]. The others symmetric modes give slightly different results. We can conclude that segregation process may be guided by different modes of transition or with mixture of mode and not only by " $2 \rightarrow 1$ " symmetric diffusion. 


\section{CRITERIA FOR THE STABILITY OF STATIONARY MICROSEGREGATED PATTERNS}

\section{A. Minimization of free energy:}

A natural criterion for stability is the minimization of free energy since $\frac{d F}{d t}<0$, however the absolute minimum is rarely reached. In a previous work [4], it has been shown that the analytic solution of the $\mathrm{CH}$ for a finite system dimension can be a good approximation for the CHO equation. This hypothesis is verified numerically as shown in 1 . This approximation enables to compute the free energy expression including the additional term of non-local interaction (Oono's term). The stable wavelength $\lambda_{s}$ in then found to scale as the power of $\alpha:$

$$
\lambda(\alpha) \sim \alpha^{1 / 3}
$$

Another approach uses solutions from direct simulation (with fixed $\alpha$ ) for small system of wavelength $\lambda_{\mathrm{CHO}}$. Starting with only two domains and two interfaces, we vary $\lambda$ in such a way that the system can dissipate the perturbation due to the small expansion $\lambda$ and relax to a steady state. This approach allow us to follow the free energy as function of the period. The steady state for a given $\alpha$ corresponds to the lowest energy reached $F_{\alpha}(\lambda)$.

It is clear that for $\alpha=0$, no minimum is attain. The free energy decreases as a function of the wavelength, leading to an unconditioned coalescence, i.e. full segregation. This result is verified analytically since the exact solution is known; the free energy is strictly decreasing and converge to 0.25 . When $0<\alpha<1 / 4$ it seems that the free energy reach a unique minimum in $\lambda$, that corresponds to the steady state as can be seen for $\alpha=10^{-5}$ in Fig ??. In fact, the variation of non-local term in the $\mathrm{CHO}$ equation (which represents the long range interaction) becomes equal to variation the short range interaction term at this precise period, which characterized the steady state.

The minimization of free energy enables to plot this period as a function of $\alpha$ in Figure 9. 


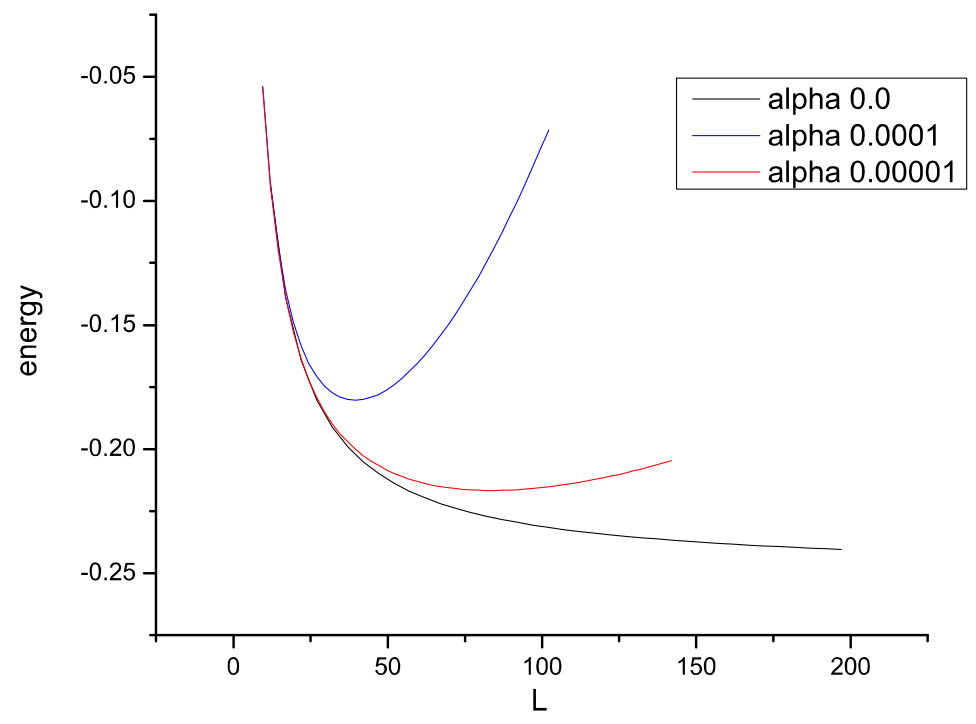

FIG. 8: The black curve illustrates the free energy as function of wavelength $\mathrm{L}$ for $\alpha=0$, red curve for $\alpha=10^{-5}$, and blue curve for $\alpha=10^{-4}$

\section{B. Misbah-Politi criterion:}

Misbah and Politi have proposed a criterion for the linear stability/instability of frozen solutions [2]. The basic idea is to perturb the periodic steady state $u_{0}(x, \lambda)=u_{0}(x+\lambda, \lambda)$ for which one can define the phase $\phi(x)=q x$ where $q=2 \pi / \lambda$, by adding some perturbation on $u_{0}: u(x, t)=u_{0}(x)+\epsilon u_{1}(x, t)$. First they rescaled $x$ by introducing slow space variable $X=\epsilon x$ and consequently slow time variable $T=\epsilon^{2} t$, and a slow phase defined as $\Psi(X, T)=$ $\epsilon \phi(x, t)$.

Keeping only first order terms in $\epsilon$ when developping the partial time/space derivatives in $x$ and $t$ in $\mathrm{CHO}$ equation as functions of $X$ and $T$ and using the fact that the stationary solution $u_{0}$ satisfies the zero order equation, they found at the first order the following phase diffusion equation

$$
\begin{aligned}
\epsilon \partial_{T} \Psi & =D \partial_{X X}^{2} \Psi \\
\text { where } \quad D & =q^{2} \frac{\partial_{q}<q\left(\partial_{\phi} u_{0}\right)^{2}>-\alpha . \partial_{q}<q w^{2}>}{<u_{0}^{2}>}
\end{aligned}
$$

This diffusion coefficient is proposed as criterion for dynamic stability. If the phase 


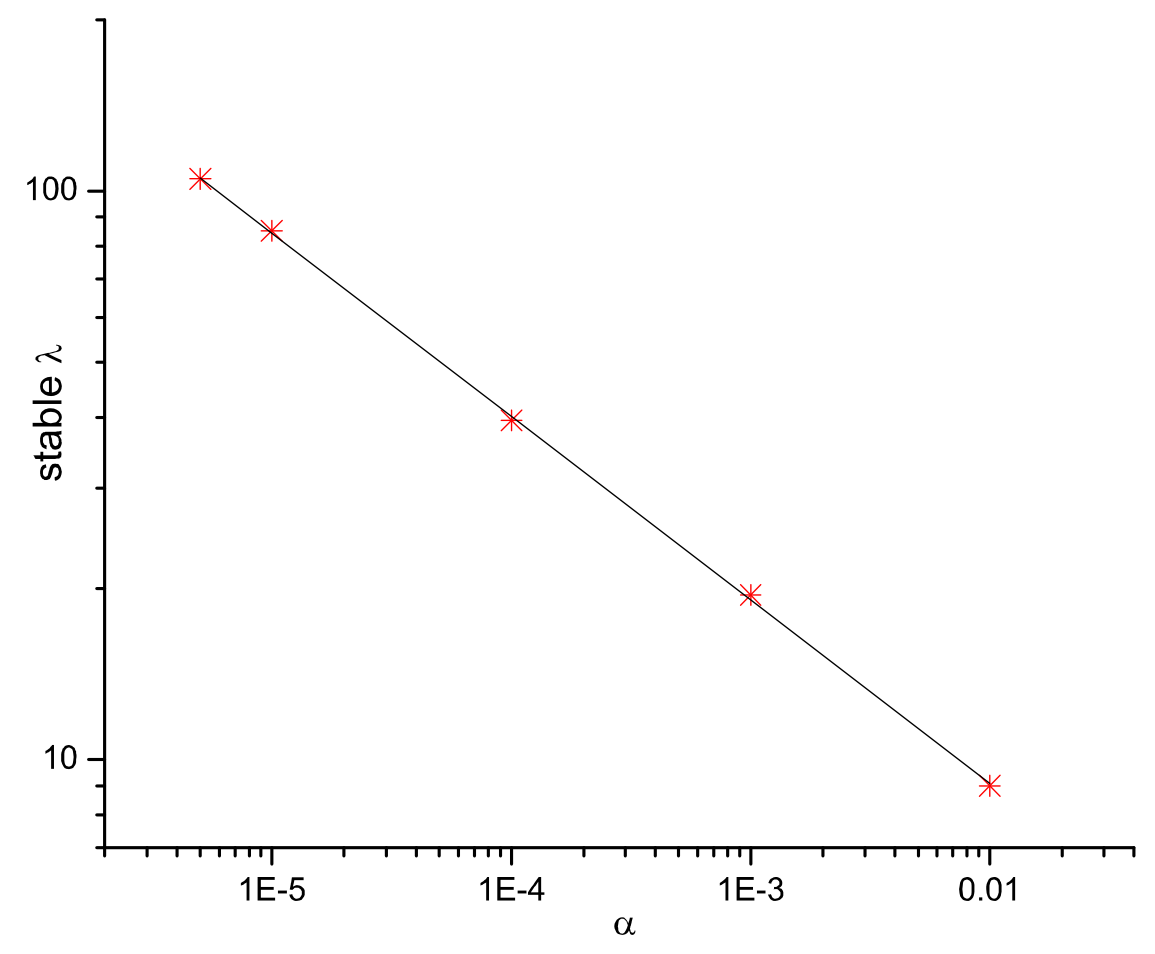

FIG. 9: In red asterix, stable wave length found by minimizing of the free energy with respect to the size of the final domains. The results scale like $\alpha^{-1 / 3}$

perturbations vanishes $(D<0)$, the stationary solution $u_{0}$ is dynamically stable ; otherwise $(D>0)$, the perturbation grows and the system will coalesce.

\section{STATISTICAL STUDY OF THE CAHN_HILLIARD EQUATION}

In this section we look at the results of direct simulation of $\mathrm{CHO}$ dynamics in order to guide our study. Since we have shown beloow that the two criteria of stability, one based on the minization of the free energy and the other based on the dynamics, give different final wave lengths, it is important to determine which one is the closest of the direct simulation result.

The relevant stationary solutions can be found only if the length of the system is larger 
than the natural wavelength. We therefore consider a large system $\left(256 \times \lambda_{C H}\right)$, for which the dynamics of pattern formation is followed for long time simulation. The spatial step is declared in terms of $\lambda_{C H}$ (256 point per $\left.\lambda_{C H}\right)$, the time step is dynamically changed to get more precision and weak white noise is added to speed up the dynamics (annealing). The wave lengths of final solutions are found by measuring the zeros of final profiles, and they are classified in small intervals of wavelength. We have focused on totally random initial conditions (of low amplitude $<0.01$ ), so we don't privilege a priori any particular solution.

In the picture 10 we show some distributions of wavelength of the final stable solution for different values of $\alpha$.

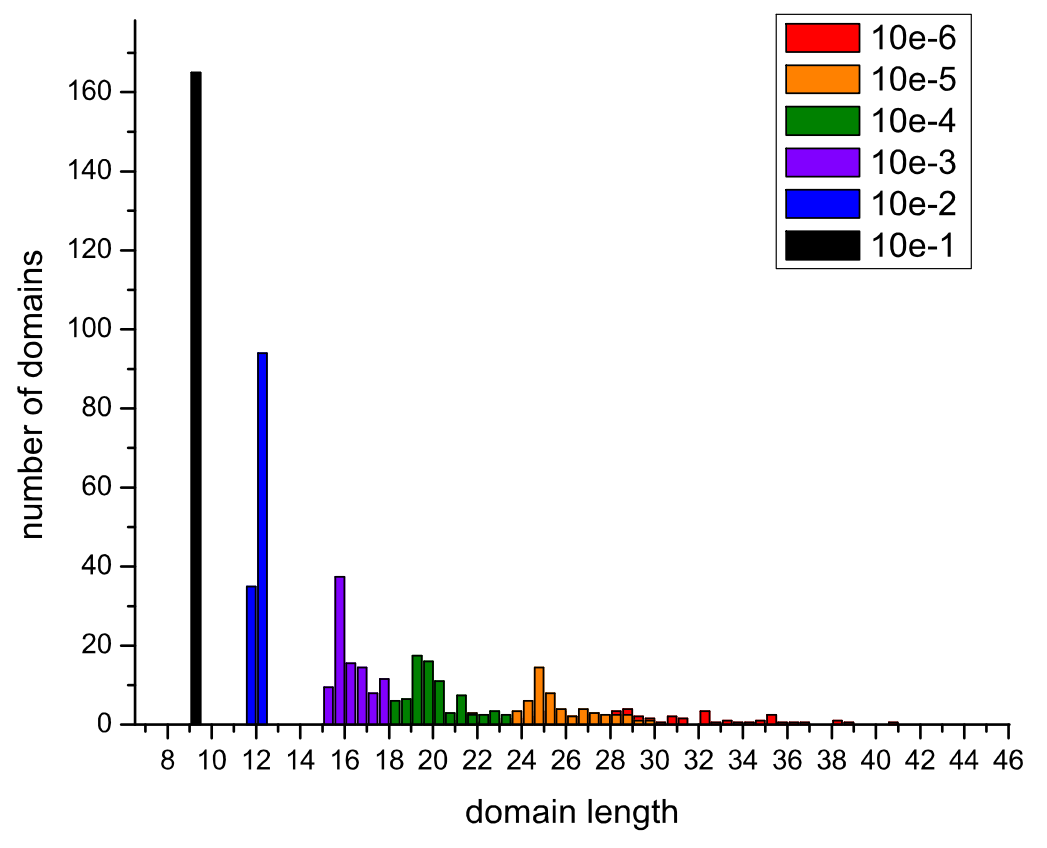

FIG. 10: In black, unique wavelength for $\alpha=0.1$, in blue, distribution of stable wavelengths for $\alpha=10^{-2}$, in violet, for $\alpha=10^{-3}$, in green for $\alpha=10^{-4}$, orange for $\alpha=10^{-5}$, and red for $\alpha=10^{-6}$

We can notice that, for high values of $\alpha$, there is only a unique wavelength ( we noticed that the final solution is obtained quickly, the final wave length is close to $\lambda_{C H}$ ). And while the distribution is becoming larger for smaller $\alpha$, we still notice the presence of a central pick value for the most probable wavelength. 


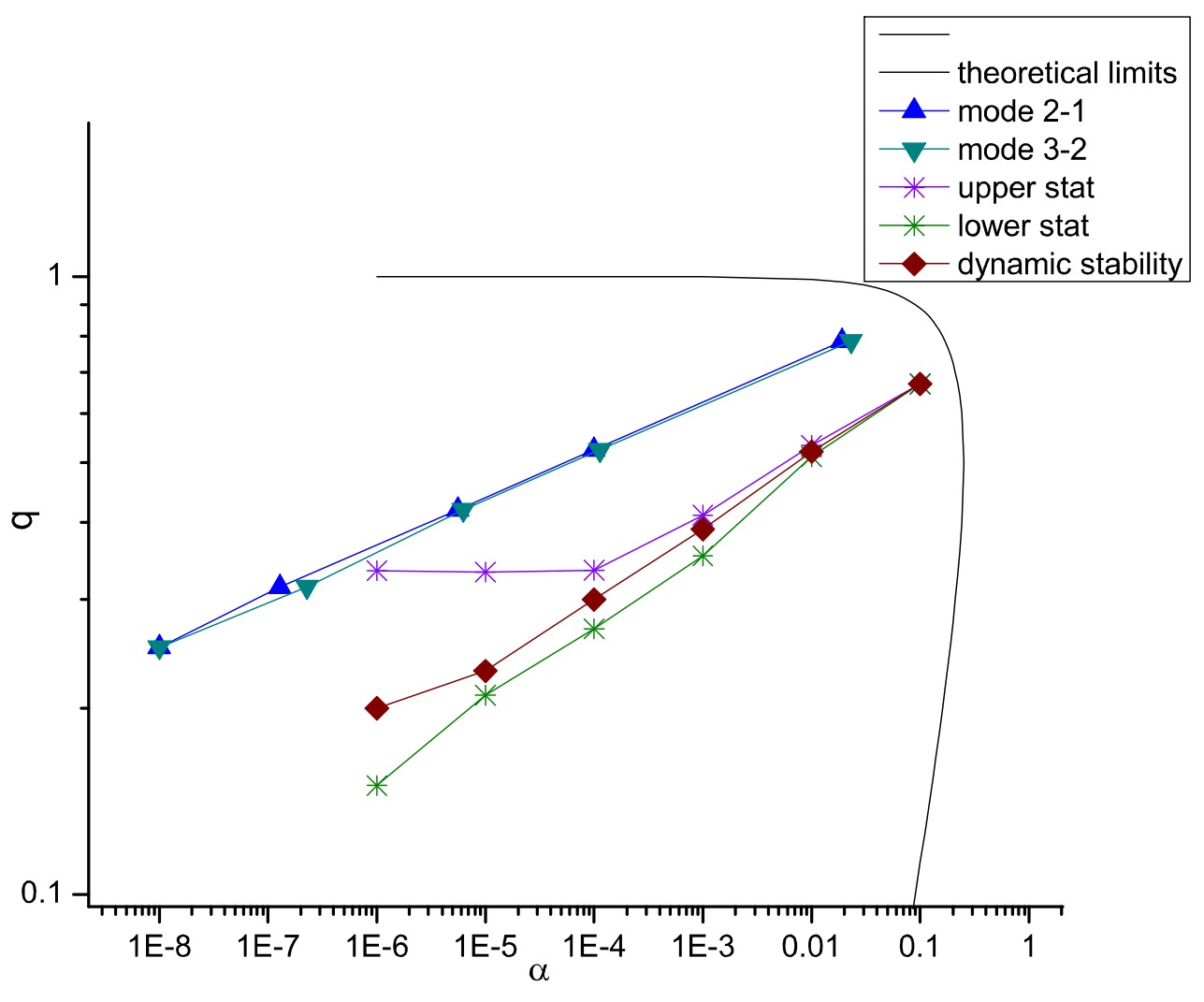

FIG. 11: Stability diagram $(q, \alpha)$ plotted in log-log to be compared to Politi-Torcini results [5] : in black the stability zone defined by the solution of $\left(1-q^{2}\right) q^{2}-\alpha=0$, in red wine, the stable wavelengths found using Misbah-Politi criterion, the blue-triangles and dark-cyan-triangles lines represent the stable wavelengths found respectly for transition modes $2 \rightarrow 1$ and $3 \rightarrow 2$, the upper and lower limits of stable solution found statically for large system using totally random initial states are showed in violet and green line-asterix.

We resume all results obtained from criteria discussed above in this picture 11 


\section{CONCLUSION}

We have studied numerically a system of large dimension, with the goal of finding a good stability criterion ending Cahn-Hilliard-Oono dynamics, as solutions are not unique and wavelengths may not be the same in different spatial part of system. In the first part, we have tried to study transition modes and how patterns evolve in there vicinity. We have found that pure symmetric diffusion process between identical domains defines the upper limit stability of $q$ or the smallest final wavelength. So we think that more complicated transition modes may be responsible for the creation of larger patterns. In the last part, we have studied statistically a large dimension system and we have compared two different approaches of stability to these statical results. We have found a region of stable wavelengths that fits very well with the stability criterion proposed by C. Misbah and P. Politi. This criterion is more appropriate to predict the final stage of the dynamics than the sole free energy minimization. However it cannot explain the distribution of solution around the central line of stability.

[1] "Coarsening dynamics in one dimension: the phase diffusion equation and its numerical implementation " M. Nicoli, P.Politi and C. Misbah, Phys. Rev. E 87, 063302 (2013).

[2] "When does coarsening occur in the dynamics of one-dimensional fronts?" P.Politi and C. Misbah, Phys. Rev. Lett. 92, 090601 (2004).

[3] "Free Energy of a Nonuniform System. I. Interfacial Free Energy " J.W. Cahn et J.E. Hilliard, J. Chem. Phys. 28, 258 (1958).

[4] "Coalescence in the 1D Cahn-Hilliard model" S. Villain-Guillot, J. Phys. A: Math. Gen. 37, 6929 (2004).

[5] "Dynamics versus energetics in pase separation"P. Politi and A. Torcini J. Stat. Mech. P03016 $(2015)$

[6] "Stability of frozen waves in the Modified Cahn-Hilliard model " E. S. Benilov,W. T. Lee, and R. O. SedakovPhys. Rev. E 87, 032138 (2013) 